\title{
The pitfalls in the development of biologic therapy
}

\author{
Ravinder N Maini and Marc Feldmann
}

'Bench to bedside' has become the mantra for researchers who wish to translate advances in knowledge and technology into tangible benefits for patients. Monoclonal antibodies and recombinant fusion proteins have become major players in the development of new, 'knowledgebased', targeted drugs - not only for rheumatoid arthritis (RA), but for other immune-mediated inflammatory diseases as well. Here we discuss some of the pitfalls and lessons learned from our experiences in this field.

The most obvious pitfall in the development of any new drug is that there is 'many a slip 'twixt the cup and the lip'. The failure of the majority of promising preclinical concepts in clinical trials testifies to this. In the case of the regulation of the cytokine network and inflammation by antibodies to tumor necrosis factor (TNF), it was the cumulative force of several preclinical in vitro and in vivo observations in human and animal models that was necessary and essential for a successful outcome in the clinic (Feldmann M and Maini RN [2003] Nature Med 9: 1245-1250).

In the late 1980s, our enthusiasm for TNF as a therapeutic target received less than warm support from some of the peer-reviewers of our grant applications. For us, however, the most painful pitfall proved to be our failure to convince representatives of the pharmaceutical industry to make existing biologic TNF antagonists available for chronic disease indications. The notion that targeting TNF alone could be effective or economically viable as a long-term therapy for RA was greeted with incredulity and skepticism. Ultimately, it was the support of a Chief Scientist in the biotechnology industry, who was a personal friend, that permitted the first clinical trial of a chimeric antibody to TNF "the vested

interests

and preset

agenda of

the influential

players in

industry can

be a serious

barrier for

the academic

researcher to

surmount'

RN Maini, previously Head of

Kennedy Institute

of Rheumatology, is

now the Emeritus

Professor of

Rheumatology, and

$M$ Feldmann is the

current Head of the

Kennedy Institute

of Rheumatology,

at Imperial College

London, London, UK.

\section{Competing interests}

The authors have declared associations with the

following companies:

Abbott, Centocor, Schering-

Plough, and Wyeth. See the

article online for full details

of the relationships.

www.nature.com/clinicalpractice doi:10.1038/ncprheum0373 in RA and the subsequent rapid development of this and two other anti-TNF biologics. The lesson learned was that the vested interests and preset agenda of the influential players in industry can be a serious barrier for the academic researcher to surmount.

Since the first patient with RA was exposed to TNF antagonists in 1992, over 1 million patients have been treated with TNF antagonists, and clinical indications have expanded to include ankylosing spondylitis, psoriatic arthritis, psoriasis and inflammatory bowel diseases. Concerns about the risks of serious infections and cancer as a consequence of suppression of host defense mechanisms by TNF blockade are being addressed by ongoing research (Dixon WG et al. [2006] Arthritis Rheum 54: 2368-2376; Setoguchi S et al. [2006] Arthritis Rheum 54: 2757-2764). The emerging consensus is that attributing a causal relationship is not straightforward, as aging patients with severe RA who have never been exposed to TNF antagonists have an increased risk of serious infections and cancer, especially lymphoma, anyway; this risk is possibly related to biologic mechanisms underlying disease pathogenesis. The picture is further complicated by the complex interplay of the potentiating or protective actions of nonbiologic disease-modifying antirheumatic drugs and corticosteroids. Despite the reassurance that TNF blockade is safer than initially thought, use of these agents in clinical practice still requires vigilance, to ensure that benefit exceeds harm. Last, but not least, access to all biologics is inequitably restricted by the ability of the purchaser to meet the high cost of the drugs; consequently, patients are often denied access to a useful therapeutic option. 